\title{
A Comparative Investigation Between Transmission Kikuchi Diffraction (TKD) and Precession Electron Diffraction (PED)
}

Glenn Sneddon ${ }^{1}$, Xuyang Zhou ${ }^{2}$, Gregory Thompson ${ }^{2}$ and Julie Cairney ${ }^{1}$

${ }^{1}$ The University of Sydney, Sydney, New South Wales, Australia, ${ }^{2}$ University of Alabama, Tuscaloosa, Alabama, United States

Transmission Kikuchi Diffraction (TKD) in the SEM [1] and Precession Electron Diffraction (PED) in the TEM [2] are methods used to achieve higher spatial resolution for grain misorientation and grain size mapping as well as grain texture quantification. Using equivalent samples, a comparison of these techniques to these types of grain characteristic measurements has been undertaken.

In a nanocrystalline $\mathrm{Cu}$ specimen, the TKD analysis indexed more grains with a higher confidence than PED, Figure 1(a). The origin of the difference is explained by how the diffraction patterns are captured. In TKD, the diffraction events are derived from within a few to ten nanometers of the exiting surface. In this case, the diffraction is then limited (typically) from a single grain that satisfies the Kikuchi diffraction condition even if other scattering events may have occurred above the exiting grain. In PED, the diffraction events are an integration through the entirety of the foil, thus the collected patterns can be a convolution if more than one grain's diffraction pattern overlaps. Nonetheless, when the grains did not overlap, PED's spatial resolution for mapping the smallest grains was more evident as compared to TKD.

Though both methods could index twin structures in the $\mathrm{Cu}$ foil, PED appears to be more sensitive to identifying twin structures via its template-matching algorithm than equivalent twins captured by TKD, as shown by the circled regions with the solid arrows in Figure 1(a). This could be a result of the inclination plane of the twin coupled with shared bands between the twin and parent yielding incorrect indexing in the TKD condition. Interestingly, when twins were close to a $\{001\}$ zone axis of a grain, PED did not reveal the twin but was observed for TKD, i.e. the dashed arrow in the boxed region in Figure 1(a).

A comparison of the pattern collection as a function of foil thickness was also undertaken. TKD was able to index diffraction patterns in foils up to $\sim 350 \mathrm{~nm}$ in thickness whereas PED pattern recognition was limited to $\sim 140 \mathrm{~nm}$ for the $\mathrm{Cu}$ foil, Figure 1(b). Though the TKD scans were done at $20 \mathrm{keV}$ and the PED at $200 \mathrm{keV}$, suggesting a peculiar contradiction in that higher $\mathrm{keV}$ would enable deeper electron penetration, the difference in pattern detection as a function of foil thickness is explained again in the origins of the diffraction events themselves. TKD patterns originate from inelastic scattered electrons that satisfy the Bragg condition for electrons exiting the bottom surface of the foil. If these electrons are able to escape the foil in this diffraction condition to yield a sufficient detected intensity, they will provide a TDK pattern. In contrast, PED patterns originate from pseudo-kinematical scattered electrons. If these electrons undergo multiple scattering events with continual penetration into the volume of the material, their ability to satisfy the Bragg condition is lost and no pattern is detected.

In summary, both TKD and PED provide complementary means of quantifying nanoscale grain characteristics. Rather than being competitive techniques to each other, each method has particular strengths and weaknesses dependent on the sample type, foil thickness, and spatial resolution needed [3]. 

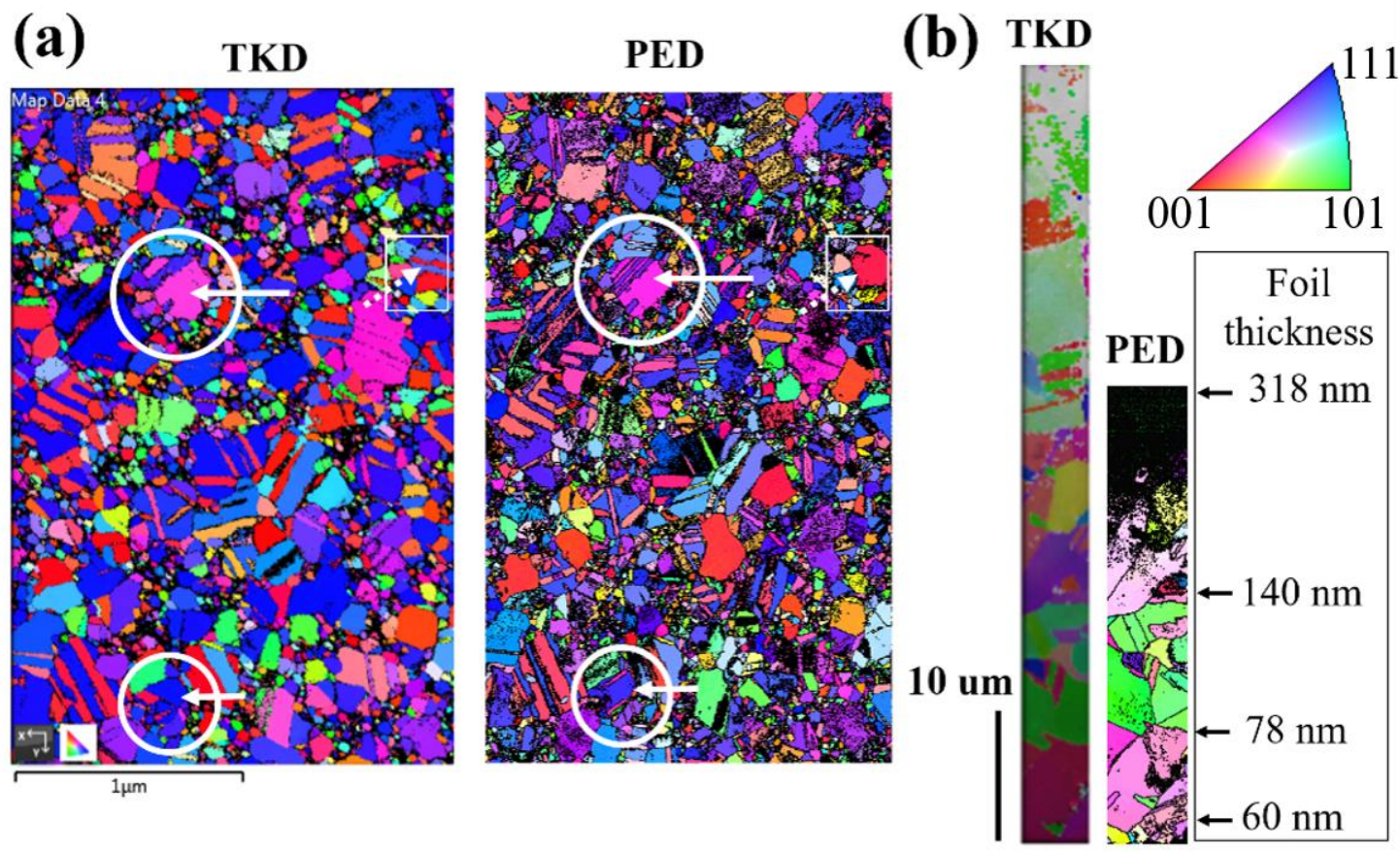

Figure 1. (a) TKD and PED map of a nanocrystalline $\mathrm{Cu}$ foil. The circled and solid arrows identify regions where PED revealed a higher spatial detection of twins whereas the boxed and dashed arrow identifies a region where TKD identified a twin that is absent in the PED scan. Each scan was done at a $5 \mathrm{~nm}$ step size. (b) A profile of collected $\mathrm{Cu}$ grains from the same region between TKD and PED as a function of foil thickness (measured by EELS). Note the further detection of the patterns for the TKD for thicker foils.

\section{References}

[1] G.C. Sneddon, P. W. Trimby, and J. M. Cairney "Transmission Kikuchi diffraction in a scanning electron microscope: A review" Materials Science and Engineering R: Reports Volume 110, December 2016, Pages 1-12

[2] P.A. Midgley and A. S. Eggeman "Precession electron diffraction - a topical review" IUCrJ Volume 2(a), January 2015, Pages 126-136

[3] The authors thank B. Britton for helpful technical discussions. XZ and GBT recognize support under NSF-DMR-1709803 for this work. 